\title{
Factores de riesgo de dislipidemia en niños y adolescentes con obesidad
}

\author{
Enrique Romero-Velarde, Dr en C, $(1,2,3)$ Octavio Campollo-Rivas, $\mathrm{PhD},{ }^{(4)}$ \\ Alfredo Celis de la Rosa, Dr en E, ${ }^{(2)}$ Edgar MVásquez-Garibay, Dr en $C,{ }^{(1,2)}$ \\ Juan Francisco Castro-Hernández, M Esp, ${ }^{(3)}$ Rosa M Cruz-Osorio, M Esp. ${ }^{(3)}$
}

\section{Romero-Velarde E, Campollo-Rivas O, Celis de la Rosa A, Vásquez-Garibay EM, Castro-Hernández JF, Cruz-Osorio RM. Factores de riesgo de dislipidemia en niños y adolescentes con obesidad. Salud Publica Mex 2007;49:103-108.}

\section{Resumen}

Objetivo. Evaluar el riesgo de dislipidemia asociado a obesidad en niños y adolescentes. Material y métodos. En un estudio transversal analítico se incluyeron 62 niños y adolescentes obesos (IMC > centila 95 y pliegue cutáneo tricipital > centila 90) y 70 no obesos (IMC centilas 5-85), con edades de 5 a 15 años, sin enfermedades crónicas. Se investigaron características personales y antecedentes familiares de enfermedades crónicas y se determinó el perfil sérico de lípidos. Se calculó el riesgo de presentar alteraciones en el perfil sérico de lípidos en niños con obesidad mediante la razón de momios (RM) y se realizó análisis multivariado. Resultados. La edad promedio para todo el grupo fue de $9.8 \pm 2.7$ años; 63 individuos eran del sexo femenino y 69 del masculino. La presencia de obesidad se asoció a riesgo de valores anormales de colesterol, triglicéridos, LDL, HDL y dislipidemia ( $>$ I valor anormal) (RM 4.47-I5.0). En obesos el análisis multivariado mostró que la pertenencia al sexo femenino se asoció significativamente a dislipidemia. Conclusiones. La obesidad en niños y adolescentes se asocia a riesgo elevado de presentar dislipidemia; este riesgo es mayor en las mujeres.

Palabras clave: obesidad; niños; adolescentes; dislipidemia; colesterol sérico; México
Romero-Velarde E, Campollo-Rivas O, Celis de la Rosa A, Vásquez-Garibay EM, Castro-Hernández JF, Cruz-Osorio RM. Risk factors for dislypidemia

in obese children and adolescents.

Salud Publica Mex 2007;49:103-108.

\section{Abstract}

Objective.To assess the risk of dislypidemia associated with obesity in children and adolescents. Material and Methods. A cross sectional study was conducted with 62 obese children (BMI > 95 centile and tricipital skinfold thickness $>90$ centile) and 70 non-obese children (BMI 5-85 centile) ages 5 - I5 years, without chronic diseases. Subjects' characteristics and family background of chronic diseases were collected and a lipid profile was determined. The risk of lipid alterations in the obese children was calculated using odds ratio (OR) and multivariate analysis. Results. Mean age was $9.8 \pm 2.7$ years in both groups; 63 girls and 69 boys were included. Obesity was associated with abnormal values for cholesterol, triglycerides, LDL, HDL and dislypidemia (> I abnormal value) (OR 4.47-I5.0). In obese children and adolescents the multivariate analysis showed that female gender was associated with dislypidemia. Conclusion. Obesity in children and adolescents is associated with high risk of dislypidemia; the risk is higher among females.

Key words: obesity; children; adolescents; dislypidemia; serum cholesterol; Mexico

(I) Instituto de Nutrición Humana, Departamento de Reproducción Humana, Crecimiento y Desarrollo Infantil.

(2) Centro Universitario de Ciencias de la Salud de la Universidad de Guadalajara.

(3) Clínica para la Atención de Niños y Adolescentes con Obesidad de la División de Pediatría del Hospital Civil de Guadalajara Dr. Juan I. Menchaca.

(4) Centro de Estudios de Alcoholismo y Adicciones, Universidad de Guadalajara.

Fecha de recibido: 24 de noviembre de 2005 • Fecha de aceptado: 13 de noviembre de 2006 Solicitud de sobretiros: Dr. Enrique Romero Velarde. Salvador Quevedo y Zubieta 750, edificio anexo al Hospital Civil Dr. Juan I. Menchaca, 3er piso, Col. Independencia. 44340 Guadalajara, Jalisco, México. Correo electrónico: erv205@hotmail.com 
$\mathbf{N}$ umerosos informes señalan que la prevalencia de obesidad en niños y adolescentes se ha incrementado notablemente en las últimas dos décadas, de tal manera que se ha convertido en un problema de salud pública..$^{1-3}$

Uno de los problemas que se han observado con el aumento en la prevalencia de obesidad en niños y adolescentes es el incremento paralelo en las complicaciones relacionadas a su presencia, entre las que se incluyen las metabólicas, las psicológicas y las ortopédicas, entre otras. ${ }^{4-6}$

Se ha descrito que los niños y adolescentes con obesidad, sobre todo aquellos con incremento en la grasa abdominal, pueden tener un perfil sérico de lípidos aterogénico, caracterizado por un incremento de la concentración de colesterol sérico total (CT), triglicéridos (TGL) y lipoproteínas de baja densidad (LDL), y por la disminución de las lipoproteínas de alta densidad (HDL); se ha llegado a notificar la presencia de estas alteraciones hasta en 30\% de niños y adolescentes con obesidad. Actualmente, se reconoce que las alteraciones del perfil sérico de lípidos en niños y adolescentes con obesidad pueden ser indicadores tempranos de riesgo cardiovascular, o formar parte del síndrome metabólico. ${ }^{7,8}$

En México, son escasos los estudios que evalúan las alteraciones del perfil sérico de lípidos en niños y adolescentes y su relación con la presencia de obesidad. Así entonces, el objetivo del presente trabajo fue, por una parte, evaluar el riesgo de presentar alteraciones en el perfil sérico de lípidos asociado a obesidad y la frecuencia de estas alteraciones, y por la otra, identificar variables asociadas a su presencia.

\section{Material y métodos}

En un estudio transversal analítico se incluyeron 62 niños y adolescentes obesos y 70 no-obesos de entre 5 y 15 años de edad, que acudieron a la consulta externa de la División de Pediatría del Hospital Civil de Guadalajara Dr. Juan I. Menchaca, entre marzo de 1997 y febrero de 2000. Se consideró la presencia de obesidad con un índice de masa corporal $($ IMC) $>$ percentil 95 y pliegue cutáneo tricipital $(\mathrm{PCT})>$ percentil 90 del patrón de referencia del National Center for Health Statistics (NCHS). ${ }^{9}$ Los criterios de inclusión fueron: no haber presentado enfermedades infecciosas en los últimos 15 días ni ser portadores de enfermedades crónicas (asma bronquial, diabetes mellitus, etc.) y que los sujetos procedieran de la Zona Metropolitana de Guadalajara. Para el cálculo del tamaño de la muestra se consideró la frecuencia de presencia de hipercoles- terolemia en niños obesos $(\sim 30 \%)$ y el riesgo $(R M=3.6)$ informado para presentar CT elevado (> $200 \mathrm{mg} / \mathrm{dL})$ en niños y adolescentes de 5 a 17 años.,10,11 Los pacientes se identificaron en la consulta externa de Pediatría, donde se obtuvo el consentimiento por escrito de sus padres. El grupo de niños y adolescentes no-obesos se seleccionó entre aquellos que acudieron a atención por padecimientos menores (p.e.: oculares o dermatológicos menores), o que se encontraban acompañando a un familiar en la consulta externa: los criterios de inclusión para este grupo fueron semejantes a los de niños obesos, pero con IMC entre el percentil 5 y 85 del mismo patrón de referencia. Los criterios de exclusión fueron no acudir a la toma de muestra sanguínea para la determinación del perfil sérico de lípidos, y la sospecha de una probable patología orgánica durante la exploración física.

Como variable dependiente se consideró la presencia de dislipidemia de acuerdo con las alteraciones de las variables que conforman el perfil sérico de lípidos, considerando como límites superiores (inferior en el caso de HDL) los recomendados por la Academia Americana de Pediatría y el Programa Nacional de Educación en Colesterol de los Estados Unidos de América (EUA) (CT > $200 \mathrm{mg} / \mathrm{dL}$; LDL >130 mg/dL; HDL $<35 \mathrm{mg} / \mathrm{dL}$ y TGL >150 mg/dL ${ }^{12,13}$ y, como variable independiente, la presencia de obesidad. Como variables de confusión se incluyeron el género, los antecedentes familiares de enfermedades crónicas (diabetes mellitus tipo II, hipercolesterolemia, infarto al miocardio, obesidad) y los grupos de edad (escolar: 5-10 años; adolescente: 11-15 años). Los antecedentes familiares de enfermedades crónicas se obtuvieron de forma verbal en la entrevista hecha a los padres y se consideraron positivos únicamente si la enfermedad había sido diagnosticada por un médico en una institución de salud. Una vez que el paciente fue incluido, se realizó una entrevista al sujeto de estudio y a sus padres, para conocer sus características y antecedentes familiares de enfermedades crónicas. Previa estandarización, ${ }^{14}$ se realizaron las mediciones antropométricas (peso, talla, circunferencia del brazo, pliegue cutáneo tricipital y subescapular) y se calcularon los índices peso/edad, peso/talla, talla/edad e IMC $\left(\mathrm{kg} / \mathrm{m}^{2}\right)$. Después, se citó a los niños y adolescentes para el día siguiente por la mañana, en ayuno (12 horas) para la toma de una muestra sanguínea en la que se determinó la concentración sérica de lípidos. La concentración sérica de CT y TGL se llevó a cabo por métodos enzimáticos (bioMériux Laboratories Vitek. Inc. ${ }^{\mathrm{TM}}$ ); HDL por precipitación en un autoanalizador de química clínica (CX-7 Beckman) y las LDL se calcularon según la 
fórmula de Friedewald. ${ }^{15}$ Se citó a los sujetos de estudio un mes después, para conocer los resultados de laboratorio.

Se realizaron estadísticas descriptivas, medidas de frecuencia, porcentaje, medidas de tendencia central y dispersión de todas las variables. Se calculó el riesgo (RM) de presentar valores elevados de CT, LDL y TGL o disminución de HDL de acuerdo con la presencia o ausencia de obesidad. Más adelante y exclusivamente con el grupo de niños y adolescentes con obesidad, se contrastaron las características generales, antropométricas y antecedentes familiares de enfermedades crónicas en niños y adolescentes con y sin dislipidemia (alteraciones simultáneas en dos o más variables del perfil de lípidos). Los índices antropométricos talla/edad (T/E) e IMC se expresaron en dos categorías elegidas en forma arbitraria para su análisis: T/E mayor o menor a 0.0 en puntuación Z e IMC mayor o menor a +3.0 desviaciones estándar (puntaje Z). Se identificaron aquellas variables con asociación significativa y valores de $R M$ superiores a la unidad, para incluirlas en modelos de regresión logística e identificar variables asociadas a riesgo de dislipidemia en niños y adolescentes con obesidad. El análisis estadístico se realizó con el programa SPSS versión 10.0. El estudio recibió la aprobación del Comité de Etica del Hospital.

\section{Resultados}

Se incluyeron 150 sujetos: 71 en el grupo de obesos y 79 en el grupo no-obesos; se excluyó a nueve sujetos de cada grupo debido a que no acudieron a la toma de la muestra sanguínea para la determinación de lípidos; sus características generales (promedios de edad e IMC y distribución por sexo) no fueron diferentes de aquellas de los que sí acudieron a la toma de la muestra. El promedio de edad para el grupo de estudio fue de $9.8 \pm 2.7$ años; 63 eran del sexo femenino y 69 del masculino, y no hubo diferencias significativas al comparar obesos contra no-obesos. El promedio de edad de los padres para los dos grupos fue de 39.6 años \pm 7.3 y de las madres de $36.1 \pm 6.0$ años, con escolaridad de $8.8 \pm 4.0$ y $7.4 \pm 3.1$ años respectivamente, ingreso familiar mensual de $2886.22 \pm 2016$ pesos (límites 50012000 pesos) y número de hijos de $3.5 \pm 1.75$, sin diferencias entre grupos. Los antecedentes familiares de infarto al miocardio ( 35.5 vs $20 \%$; $p<0.05$ ) y obesidad (87.1 vs $44.3 \%$; $p<0.001$ ), fueron más comunes en niños y adolescentes con obesidad; los antecedentes de hipercolesterolemia y diabetes mellitus tipo II no mostraron diferencias entre grupos. En el grupo de obesos, $87.1 \%$ informaron antecedente positivo para obesidad en la familia; la frecuencia de menciones para el padre fue de 33 ocasiones (53.1\%), la madre de 38 (60.9\%) y algún hermano de $12(20.3 \%)$. El resto de las menciones fueron para abuelos, tíos o primos por ambas ramas; los familiares de rama materna fueron citados más frecuentemente que los de rama paterna. En el cuadro I se muestran los valores promedio de los índices antropométricos peso/edad, peso/talla, talla/edad e IMC (expresados en puntuación Z), así como de los pliegues cutáneos para los dos grupos.

El promedio de la concentración sérica de CT y TGL fue superior en niños con obesidad $(<0.01)$, mientras que el promedio de la concentración de HDL fue inferior $(<0.001)$; no hubo diferencias en la concentración de LDL entre grupos (cuadro II). En el cuadro III se muestra el riesgo significativo de presentar alteraciones en el perfil sérico de lípidos y dislipidemia en niños y adolescentes con obesidad; el valor de la $R M$ más elevado fue para dislipidemia $(R M=15.02$; IC $95 \%$ 3.13-98.5).

Al analizar por separado a los niños con obesidad y comparar el comportamiento de algunas variables entre los que presentaron $(n=19)$ o no $(n=43)$ dislipidemia, se observó que la pertenencia al sexo femenino $(R M=4.04, I C 95 \% 1.28-12.8)$, la edad adolescente $(R M=$ 3.20, IC 95\%1.04-9.8) y el índice T/E ( $<0.0$ en puntuación Z) ( $R M=3.20$, IC 95\%1.04-9.8), se asociaron con mayor riesgo de dislipidemia. Paradójicamente, el grupo con dislipidemia tuvo una frecuencia significativamente menor de antecedente de infarto en la familia (cuadro IV). Con estas variables se construyó un mo-

\section{Cuadro I}

\section{EdAD E ÍNDICES ANTROPOMÉTRICOS EN NIÑOS Y ADOLESCENTES OBESOS Y NO-OBESOS.} Guadalajara, Jalisco, Marzo I997-Febrero 2000

\begin{tabular}{|c|c|c|c|}
\hline Variable* & Obesos $(n=62)$ & No obesos $(n=70)$ & P\# \\
\hline Edad (años) & $9.8 \pm 2.6$ & $9.7 \pm 2.9$ & 0.84 \\
\hline Peso / Edad & $3.55 \quad(2.2)$ & $-0.15(0.8)$ & $<0.001$ \\
\hline Peso / Talla ${ }^{\ddagger, \S}$ & $4.05 \quad(2.1)$ & $-0.11 \quad(0.8)$ & $<0.001$ \\
\hline Talla / Edad $\$, \S$ & $0.24 \quad(1.2)$ & $-0.23(1.2)$ & 0.02 \\
\hline $\mathrm{IMC}\left(\mathrm{kg} / \mathrm{m}^{2}\right)^{\ddagger, \delta}$ & $\begin{array}{ll}4.3 & (2.1)\end{array}$ & $-0.04(0.7)$ & $<0.001$ \\
\hline PCT $(\mathrm{mm})$ & $(6.4)$ & $12.3 \quad(5.2)$ & $<0.001$ \\
\hline PCS (mm) & $27.7 \quad(7.5)$ & $9.3 \quad(4.6)$ & $<0.001$ \\
\hline \multicolumn{4}{|c|}{$\begin{array}{l}\text { * Promedio y desviación estándar } \\
\text { ‡ Patrón de referencia: NCHS } \\
\S \text { Expresados en puntuación Z } \\
\text { \# } t \text { de Student }\end{array}$} \\
\hline \multicolumn{4}{|c|}{$\begin{array}{l}\text { IMC= índice de masa corporal } \\
\text { PCT y PCS= pliegues cutáneos tricipital y subescapular }\end{array}$} \\
\hline
\end{tabular}


Cuadro II

Concentración SÉRICA Del PERFIL SÉRICO DE LípIDOS EN NIÑOS Y ADOLESCENTES OBESOS Y NO OBESOS. Guadalajara, Jalisco. Marzo I 997-Febrero 2000

\begin{tabular}{|c|c|c|c|}
\hline Variable* & Obesos $(n=62)$ & No obesos $(n=70)$ & $P_{\ddagger}^{\ddagger}$ \\
\hline Colesterol total (mg/dL) & $177.6(47.1)$ & | $48.8(27.0)$ & $<0.001$ \\
\hline LDL- (mg/dL) & II 5.5 (44.6) & $107.5(26.0)$ & 0.21 \\
\hline$\overline{\mathrm{HDL}}{ }^{\S}(\mathrm{mg} / \mathrm{dL})$ & $37.0(8.2)$ & $42.5 \quad(6.2)$ & $<0.001$ \\
\hline Triglicéridos (mg/dL) & I $49.7(95.1)$ & II3.0 (40.6) & $<0.01$ \\
\hline \multicolumn{4}{|c|}{$\begin{array}{l}\text { * Promedio y desviación estándar } \\
\ddagger t \text { de Student }\end{array}$} \\
\hline $\begin{array}{l}\mathrm{LDL}=\text { Lipoproteínas de } \\
\mathrm{HDL}=\text { Lipoproteínas de }\end{array}$ & $\begin{array}{l}\text { densidad } \\
\text { densidad }\end{array}$ & & \\
\hline
\end{tabular}

delo de regresión logística con dislipidemia como variable dependiente y se observó que la pertenencia al sexo femenino no modifica su significado en el modelo; la edad adolescente y el índice $\mathrm{T} / \mathrm{E}<0.0$ pierden su valor como variables de riesgo. Se realizaron modelos similares e independientes para cada uno de los componentes del perfil sérico de lípidos, y se encontró que el comportamiento para CT y LDL fue similar al de dislipidemia, aunque los límites del intervalo de confianza para sexo femenino abarcan la unidad; en el modelo para HDL sólo "edad adolescente" estuvo asociada, al igual que "sexo femenino" en el modelo de TGL.

\section{Discusión}

Hoy en día, se reconoce que la obesidad en niños y adolescentes constituye un problema de salud pública; esos individuos pueden llegar a ser adultos obe-

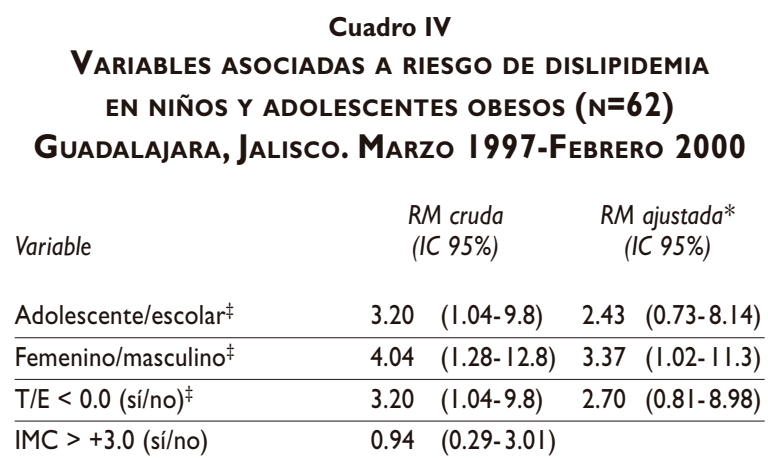

Antecedente familiar de

\begin{tabular}{lll} 
Infarto (sí/no) & 0.24 & $(0.06-0.93)$ \\
\hline Hipercolesterolemia (sí/no) & 1.51 & $(0.48-4.74)$ \\
\hline Obesidad (sí/no) & 3.50 & $(0.40-30.66)$ \\
\hline Diabetes (sí/no) & 0.51 & $(0.17-1.54)$
\end{tabular}

* Ajustados entre sí mediante regresión múltiple

$\ddagger$ ji cuadrada: $p<0.05$

$R M=$ Razón de momios

$\mathrm{T} / \mathrm{E}, \mathrm{IMC}=$ índice talla/edad e índice de masa corporal expresados en puntuación $\mathrm{Z}$

sos. Por otra parte, la obesidad en adultos se asocia con complicaciones que incrementan la morbilidad y mortalidad. ${ }^{16}$ Se ha señalado que la acumulación excesiva de grasa corporal que caracteriza a los sujetos con obesidad se asocia con cambios morfológicos y fisiológicos del tejido adiposo que condicionan la disminución de la sensibilidad a la insulina y el incremento en la lipólisis. Estos cambios se relacionan, a su vez, con resistencia a la insulina y dislipidemia. ${ }^{17}$

El presente estudio describe la frecuencia de presentación de alteraciones del perfil sérico de lípidos y

Cuadro III

Riesgo (RM) DE PRESENTAR ALTERACIONES EN LOS VALORES DEL PERFIL SÉRICO DE LÍPIDOS Y DISLIPIDEMIA EN NIÑos y adolescentes obesos Guadalajara, Jalisco. Marzo 1997-Febrero 2000

\begin{tabular}{|c|c|c|c|}
\hline Variable & $\begin{array}{c}\text { Obesos }(n=62) \\
(\text { silno })\end{array}$ & $\begin{array}{c}\text { No obesos }(n=70) \\
(\operatorname{silno})\end{array}$ & $\begin{array}{l}R M^{*}(\text { IC 95\%) } \\
\text { (silno) }\end{array}$ \\
\hline Colesterol total $>200 \mathrm{mg} / \mathrm{dL}$ & $16 / 46$ & $4 / 66$ & $5.74(1.65-21.86)$ \\
\hline $\mathrm{LDL}>130 \mathrm{mg} / \mathrm{dL}$ & $13 / 48$ & $4 / 66$ & $4.47(1.25-17.43)$ \\
\hline $\mathrm{HDL}<35 \mathrm{mg} / \mathrm{dL}$ & $24 / 38$ & $7 / 63$ & $5.68(2.07-16.17)$ \\
\hline Triglicéridos > $150 \mathrm{mg} / \mathrm{dL}$ & $24 / 38$ & $4 / 66$ & $10.42 \quad(3.1-38.57)$ \\
\hline Dislipidemia (> de I valor anormal) & $19 / 43$ & $2 / 68$ & $15.02(3.13-98.5)$ \\
\hline \multicolumn{4}{|l|}{ * Razón de momios } \\
\hline
\end{tabular}


dislipidemia en un grupo de niños y adolescentes con obesidad y su asociación con variables personales y familiares. Además, demuestra la asociación que existe entre la presencia de obesidad y este tipo de alteraciones, aunque la población de estudio se seleccionó entre los individuos que acuden a una unidad de atención hospitalaria, y tiene la ventaja de haber incluido un grupo control de niños y adolescentes sin obesidad procedentes de la misma población.

Los componentes del perfil sérico de lípidos en los que se identificaron alteraciones con mayor frecuencia fueron TGL y HDL (38.7\% cada uno); $25.8 \%$ con elevación de CT y $20.9 \%$ con elevación de LDL, cifras que concuerdan con otros informes. ${ }^{11,18}$ Por otra parte, $30.6 \%$ presentaron alteraciones simultáneas en dos o más fracciones del perfil sérico de lípidos (dislipidemia). Al evaluar la presencia de obesidad como factor de riesgo de alteraciones en el perfil sérico de lípidos, se encontraron valores crudos de RM entre 4.47 y 10.42 para las diferentes variables (cuadro III), cercanos a los notificados por Freedman en un estudio realizado en 9167 niños y adolescentes de 5 a 17 años de edad en la comunidad de Bogalusa (EUA), y que fluctuaron entre 2.4 y 7.1 para las mismas variables y el mismo criterio antropométrico de obesidad y puntos de corte del perfil sérico de lípidos (excepto TGL $<130 \mathrm{md} / \mathrm{dL}$ ), en un modelo ajustado por edad, sexo y raza. ${ }^{11}$

Cabe señalar que, en la evaluación del grupo de niños y adolescentes con obesidad, los valores crudos de la RM mostraron que el hecho de ser adolescente, pertenecer al sexo femenino y tener valores del índice $\mathrm{T} / \mathrm{E}$ por debajo de 0.0 en puntuación $\mathrm{Z}$, se asociaron a riesgo de dislipidemia con una $R M$ de 3.20 a 4.04 .

Otros estudios han informado valores superiores de lípidos en niñas (comparadas con niños) de 12 a 14 y de 6 a 15 años. ${ }^{19,} 20$ y se ha considerado que las diferencias pueden estar relacionadas con el origen étnico de la población, las diferencias culturales o de maduración sexual y la influencia de la adiposidad, que es mayor en mujeres que en hombres durante la pubertad.

Es interesante el hallazgo de que, con menor talla para la edad en niños y adolescentes con obesidad, aumenta el riesgo de dislipidemia. Una explicación a este hallazgo sería que la talla menor de este grupo de niños y adolescentes sea el resultado de desnutrición con desaceleración del crecimiento en etapas previas al desarrollo de obesidad. A este respecto, se ha descrito que tanto la desnutrición in utero, como aquella que sucede en etapas tempranas de la vida, pueden asociarse a mayor riesgo de obesidad, enfermedades crónicas y dislipidemia. ${ }^{21-23}$

El hallazgo que asocia la presencia de dislipidemia en niños y adolescentes obesos a una talla menor podría tener implicaciones que relacionan cualquier alteración que interfiera con el crecimiento lineal de un individuo en etapas previas de la vida, con mayor riesgo de presentar este tipo de alteraciones. La prevalencia de déficit de talla $(<-2 D E)$ como una expresión de subalimentación crónica (desmedro) en menores de cinco años en el país, es de $17.6 \%{ }^{3}$ Muchos niños en estas condiciones podrían desarrollar sobrepeso y obesidad en etapas posteriores, con un mayor riesgo de presentar este tipo de alteraciones y, en el largo plazo, mayores probabilidades de padecer enfermedades cardiovasculares.

Cabe destacar que el punto de corte del índice T/E utilizado en el presente trabajo (0.0 en puntuación Z) fue arbitrario, ya que el criterio para considerar a un niño con déficit de talla es por debajo de $-2 D E$ de la mediana del patrón de referencia. ${ }^{24,25}$ En este caso, no hubo niños o adolescentes con T/E por debajo de -2 $D E$ y sólo cuatro se encontraban por debajo de $-1 D E$.

Por último, se buscó identificar la asociación entre los valores superiores de IMC (en obesos) y mayor riesgo de dislipidemia. No obstante, no se encontró tal asociación; al respecto, ya se ha descrito que la proporción de niños con alteraciones metabólicas se incrementa sustancialmente conforme los valores de IMC indican mayor grado de sobrepeso. ${ }^{11}$

Se puede concluir que la presencia de dislipidemia en niños y adolescentes con obesidad es frecuente y similar a la que se indica en otros trabajos; ${ }^{11,18}$ la pertenencia al sexo femenino puede considerarse asociada a mayor riesgo de dislipidemia en este grupo de niños y adolescentes. Cabe señalar que estas alteraciones pueden persistir hasta la edad adulta y que, en el corto plazo, la reducción de peso corporal se relaciona con la mejoría de diferentes alteraciones, entre las que se encuentran aquellas del perfil sérico de lípidos.

\section{Agradecimientos}

A la Universidad de Guadalajara, al Hospital Civil de Guadalajara Dr. Juan I. Menchaca y a CONACYT-SIMORELOS, por su apoyo en la realización de esta investigación.

\section{Referencias}

I. Troiano RP, Flegal KM. Overweight children and adolescents: Description, epidemiology, and demographics. Pediatrics 1998;101: 497-504.

2. De Onis $M, B$ Bössner M. Prevalence and trends of overweight among preschool children in developing countries. Am J Clin Nutr 2000;72:1032-1039. 
3. Rivera DJ, Shamah LT,Villalpando HS, González CT, Hernández PB, Sepúlveda J. Encuesta Nacional de Nutrición 1999. Estado nutricio de niños y mujeres en México. Cuernavaca: Instituto Nacional de Salud Pública, 200I.

4. Dietz WH. Health consequences of obesity in youth: Childhood predictors of adult disease. Pediatrics 1998;10 I suppl:5 |8-524.

5. Barlow SE, Dietz WH. Obesity evaluation and treatment: expert committee recommendations. Pediatrics 1998; 102: e29.

6. Rocchini AP. Childhood obesity and diabetes epidemic. N Engl J Med 2002;346:854-855.

7. Ram W, Dziura J, Burgert TS, Tamborlane WV, Taksali SE, Sara E, et al. Obesity and the metabolic sydrome in children and adolescents. N Engl J Med 2004;350:2363-2374.

8. Jessup A, Harrell JS. The metabolic syndrome: Look for it in children and adolescents, too!. Clinical Diabetes 2005;23:26-32.

9. Frisancho RA. Anthropometric standards for the assessment of growth and nutritional status. Ann Arbor:The University of Michigan Press, 1993.

10. Mejía-Aranguré JM, Fajardo-Gutiérrez A, Gómez-Delgado A, CuevasUrióstegui ML, Hernández-Hernández DM, Garduño-Espinoza J et al. El tamaño de muestra: un enfoque práctico en la investigación clínica pediátrica. Bol Med Hosp Infant Mex 1995;52:38I-39I.

II. Freedman DS, Dietz WH, Srinivasan SR, Berenson GS. The relation of overweight to cardiovascular risk factors among children and adolescents: The Bogalusa Heart Study. Pediatrics 1999;6: I 75 - I 182. 12.American Academy of Pediatrics. Committee on Nutrition. Cholesterol in childhood. Pediatrics 1998;101:141-147.

13. National Cholesterol Education Program. Report of the Expert Panel on blood cholesterol levels in children and adolescents. Pediatrics 1992;89 suppl:525-583.

14. World Health Organization. Standarization procedures for the collection of weight and height data in the field. En:World Health Organization. Measuring change in nutritional status. Ginebra:WHO, 1983:41-45.
I5. Friedewald WT, Levy RI, Fredrickson DS. Estimation of plasma low density lipoprotein cholesterol concentration without use of the preparative ultracentrafuge. Clin Chem 1972;18:499-502.

16. Speiser WP, Rudolf MC, Anhalt H, Camacho-Hubner C, Chiarelli F, Eliakim A, et al. Consensus statement: Childhood obesity. J Clin Endocrinol Metab 2005;90:187I-I887.

17. Martin LJ,Woo JG, Daniels SR, Goodman E, Dolan ML. The relationships of adiponectin with insulin and lipids are strengthened with increasing adiposity. J Clin Endocrinol Metab 2005;90:4255-4259. 18. Dietz WH. Childhood weight affects adult morbidity and mortality. J Nutr 1998; I28:S4II-S4I4.

19. Bellu R, Ortisi MT, Scaglioni S, Agostini C, Salanitri VS, Riva E, et al. Lipid and apolipoprotein A-I and B levels in obese school-age children: Results of a study in the Milan area.J Pediatr Gastroenterol Nutr 1993;16:446-450.

20. Nain-Feng Chu, Rimm EB, Wang DJ, Liou HS, Shieh SM. Clustering of cardiovascular disease risk factors among obese schoolchildren:The Taipei Children Heart Study. Am J Clin Nutr 1998;67: I I4I-I I 46.

21. Forrester TE, Wilks RJ, Bennett Fl, Simeon D, Osmond C, Allen M, et al. Fetal growth and cardiovascular risk factors in Jamaican schoolchildren. BMJ 1996;3I2:156-160.

22. Roseboom TJ, van der Meulen JHP, Osmond C, Barrer DJP, Ravelli ACJ, Bleker OP. Plasma lipid profiles in adults after prenatal exposure to the Dutch famine. Am J Clin Nutr 2000;72:1 I0I-I I06.

23. Forsen TJ, Eriksson JG, Osmond C, Barker DJ. The infant growth of boys who later develop coronary heart disease. Ann Med 2004;36:389-392.

24. Secretaría de Salud. Norma Oficial Mexicana NOM-03I-SSA2-1999 para la atención a la salud del niño. Diario Oficial 20009 de junio: l-42. 25.WHO. Expert committee on physical status: the use and interpretation of anthropometry: Report of a WHO expert committee. WHO Technical Report Series: 854. Ginebra:WHO, 1995. 